\title{
High-resistance low-doped GaAs and AIGaAs layers obtained by LPE
}

\author{
S.I. Krukovsky, D.M. Zayachuk, O.V. Rybak, I.O. Mryhin \\ SRC "Karat", 202 Stryiska st, 290031 Lviv, Ukraine \\ Phone: (0322) 652245,e-mail: krukovsky@polynet.lviv.ua
}

\begin{abstract}
Influence of $\mathrm{Yb}$ and $\mathrm{Al}$ co-doping gallium melt during LPE growth of the GaAs epitaxial layers on their properties is investigated. It is shown that both morphology and electrophysical parameters of the films are changed under influence of the doping impurities applied. Obtained results are explained by simultaneous $\mathrm{Yb}$ gettering action with respect to oxygen and silicon in the solution-melt, and also by lowering the concentration of $\mathrm{Si}$ in the films due to $\mathrm{Al}$ entering into $\mathrm{Ga}$ sub-lattice.
\end{abstract}

Keywords: gallium arsenide, electrophysical properties

Paper received 29.12.02; accepted for publication 18.03.03.

\section{Introduction}

High-resistant low-doped GaAs and AlGaAs layers are the basis to create the fast-operating high-voltage diodes, thyristors, thermosensors and any sensors of radiation. There arise difficulties while obtaining pure III-V layers by LPE due to the presence of background impurities like silicon, carbon, oxygen, magnesium in an initial charge. Significant progress has been achieved in controlling the properties of semiconductor materials using rare-earth elements (REE) for recent 10-15 years [1-3]. Taking into account high chemical activity of the REE, the best conditions in this aspect can be potentially provided by LPE. At the same time, it is rather difficult to realise possible advantages of using rare-earth elements to control properties of GaAs and AlGaAs layers by utilising currently LPE based on the adjusting the vapour phase humidity and using quartz equipment. The main cause for that is interaction between aluminium and REE on the one hand, and quartz equipment on the other, which prevents creation of $p+-\mathrm{i}-n+$ structures in GaAs $-\mathrm{AlGaAs}$ system [4]. However, just these heterostructures attract significant practical interest due to their low back-voltage currents and higher breakdown voltages in comparison to materials with a smaller energy band gap [4,5]. In the present work, new approaches based on the complex doping with REE and isovalent element impurities to be applied to control electrophysical properties of the LPE grown GaAs layers are developed.

\section{Experimental technique}

GaAs epitaxial layers were grown by LPE method using experimental setup with horizontal reactor in the piston type cassette with growth gap of $1 \mathrm{~mm}$. Crystallisation of the GaAs layers was realised from the tin, ytterbium and aluminium doped gallium solution-melts on the semiinsulating (100)-oriented GaAs substrates with 10 arc min off-orientation towards (110) plane. With a purpose to form a charge, gallium 99.9997, aluminium 99.9999 and ytterbium 99.9 were used. The solution-melts were not preannealed. Temperature and duration of the homogenisation process were $850^{\circ} \mathrm{C}$ and 1.5 hours, respectively. Growth of layers was performed in the temperature range of $800-700{ }^{\circ} \mathrm{C}$, crystallisation velocity was $1.2^{\circ} \mathrm{C} / \mathrm{min}$. The thickness of the grown layers was normally about $15-30 \mu \mathrm{m}$. 


\section{S.I. Krukovsky et al.: High-resistance low-doped GaAs and ...}

\section{Results and discussions}

Two experimental series were performed with the purpose to investigate influence of doping $\mathrm{Ga}$ melts with $\mathrm{Yb}$ and $\mathrm{Al}$ impurities on the electrophysical properties of GaAs layers. For both series the temperature of the layer growth was the same and varied in the range of 70 to $100^{\circ} \mathrm{C}$.

For the first experimental series, Ga solution-melts were only doped with $\mathrm{Yb}$, concentration of which varied from 0.0025 to 0.038 at.\%. For the second series, Ga melt was simultaneously doped with $\mathrm{Al}$ and $\mathrm{Yb}$. In this case, Al concentration was adjusted to two fixed values equal to 0.015 and 0.026 at.\%, respectively, while $\mathrm{Yb}$ concentration varied from 0 to 0.055 at. $\%$ for each fixed value of the $\mathrm{Al}$ concentration.

Our experiments have shown that both morphology and electrophysical parameters of the films were changed under influence of the doping impurities applied. Increase in $\mathrm{Yb}$ impurity concentration may lead to worsening of the film morphology, if its value exceeds the certain critical value. Such an effect of the REE influence on the surface morphology of the films grown by LPE was known before our investigations [1]. We have found the critical concentration of the REE impurity to be dependent on the presence of other doping impurities and their nature. Thus, for example, little worsening of the films morphology become apparent when $\mathrm{Yb}$ concentration in the solution-melt exceeds 0.05 at. $\%$, whereas when solution-melt is additionally doped with $\mathrm{Al}$ impurity in amount of 0.038 at.\%, the worsening of the morphology of the growing film takes place at lower $\mathrm{Yb}$ concentration -0.04 at. $\%$.

Peculiarities of the influence of the $\mathrm{Yb}$ and $\mathrm{Al}$ impurities on the electrophysical properties of the LPE grown GaAs layers are illustrated by data shown in Figs 1 and 2 .

As one can see from Fig. 1, adding $\mathrm{Yb}$ in the Ga solution-melt decreases electron concentration (curve 1). When $\mathrm{Yb}$ concentration reaches certain critical value $N_{\text {cr }}(\mathrm{Yb})$, which is about 0.040 at. \% in our case, inversion of the conductivity type of the epitaxial layers from electronic to hole one occurs. The same behaviour of the modification of the electron concentration under $\mathrm{Yb}$ impurity influence was observed also at co-doping initial gallium solution-melts with ytterbium and aluminium (Fig. 1, curves 2 and 3). In that case, however, $N_{\mathrm{cr}}(\mathrm{Yb})$ appeared to be essentially dependent on Al concentration in the melt, namely: when the latter concentration increases, $N_{\mathrm{cr}}(\mathrm{Yb})$ decreases in comparison to the value for the layers grown from the solution-melts doped with Yb only.

Complex doping the initial solution-melts influences also the carrier mobility in the grown epitaxial layers. It reaches its maximal value approximately at the doping impurity concentrations corresponding to the points of the conductivity type inversion of the crystallising layers (Fig. 2 curves 1-3). Electron mobilities measured at 300 and $77 \mathrm{~K}$ in the GaAs layers grown from the Ga melts containing $\mathrm{Yb}$ at 0.018 at.\% concentration were found to differ almost by 7 times in their amount (Fig. 2, curves 3

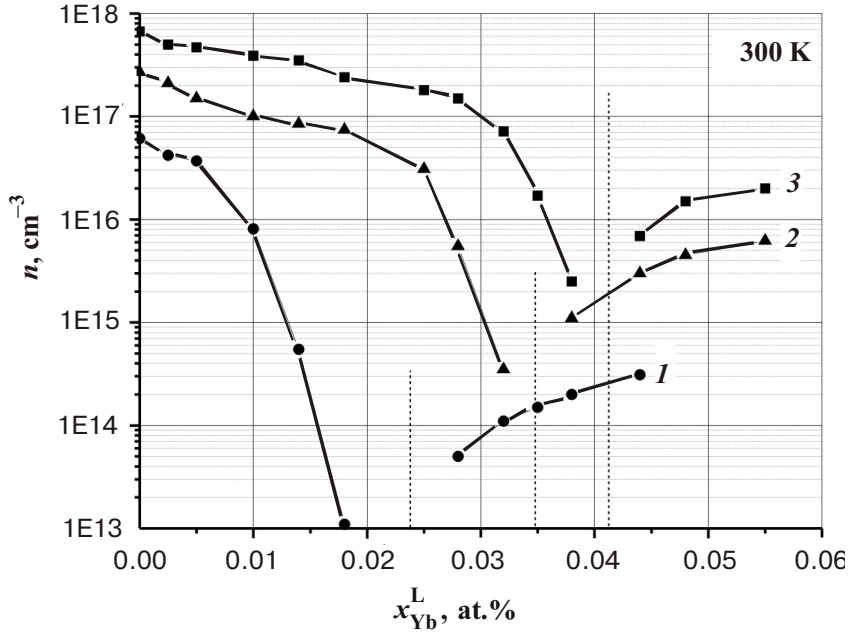

Fig. 1. Electron and hole concentrations in the epitaxial GaAs layers versus $\mathrm{Al}$ and $\mathrm{Yb}$ concentrations in the galium melt: $I-$ 0 at. $\% \mathrm{Al} ; 2-0.015$ at. $\% \mathrm{Al} ; 3-0.026$ at. $\% \mathrm{Al}$.

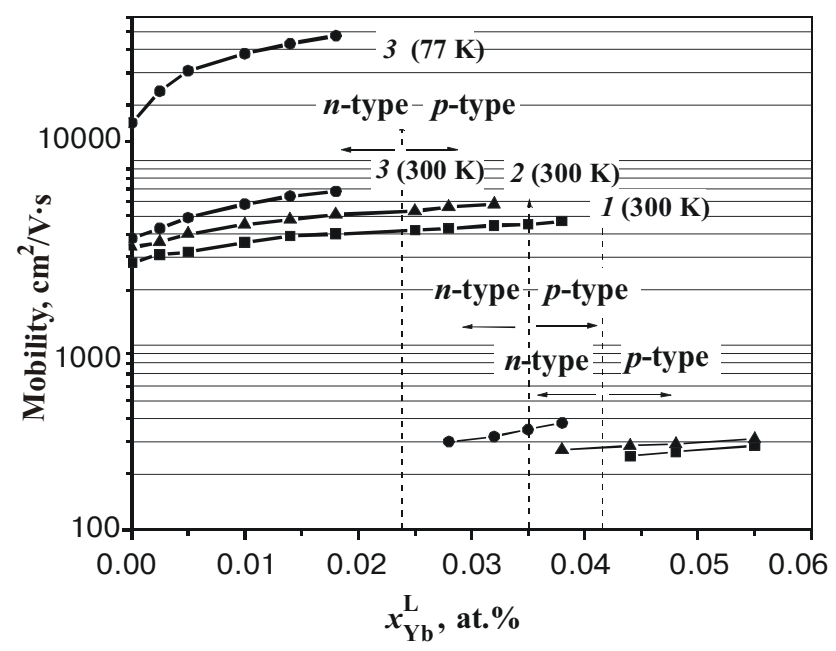

Fig. 2. Electron and hole mobilities in the epitaxial GaAs layers versus $\mathrm{Al}$ and $\mathrm{Yb}$ concentrations in the gallium melt: $1-0$ at.\% $\mathrm{Al} ; 2-0.015$ at. $\% \mathrm{Al} ; 3-0.026$ at. $\% \mathrm{Al}$.

and 4). Note that in the GaAs layers with electron concentration $1.1 \cdot 10^{16}-9 \cdot 10^{15} \mathrm{~cm}^{-3}$, the electron mobility value agrees very well with the theoretical one for the undoped films $\sim 6 \cdot 10^{3} \mathrm{~cm}^{2} / \mathrm{V} \cdot \mathrm{s}$ at $300 \mathrm{~K}$ [6]. Significant increase of the mobility at $77 \mathrm{~K}$ in comparison to that at room temperature indicates the absence of the compensation of the impurity centers in the epitaxial GaAs layers and presence of the $\mathrm{Al}$ and $\mathrm{Yb}$ induced effective gettering action in the melt with respect to the main background impurities.

A decrease in the electron concentration is accompanied by increasing the specific resistance of GaAs epitaxial layers. The maximum value of the specific resistance for the $n$-type layers, grown from the Ga melts doped with $\mathrm{Yb}$, is $10-20 \mathrm{Ohm} \cdot \mathrm{cm}$. In the layers co-doped 


\section{S.I. Krukovsky et al.: High-resistance low-doped GaAs and ...}

with $\mathrm{Yb}$ and $\mathrm{Al}$ for their concentrations in the melt equal to 0.032 and 0.015 at. $\%$, respectively, the specific resistance increases up to $5 \cdot 10^{5} \mathrm{Ohm} \cdot \mathrm{cm}$. Increasing $\mathrm{Al}$ content in the melt causes further increasing in the specific resistance of the films. It is important to note that the same value of the specific resistance of the layers can be reached at sufficiently lower $\mathrm{Yb}$ concentration by increasing $\mathrm{Al}$ concentration in the melt.

Observed behaviour of the electrophysical parameters of GaAs layers obtained from Ga melts co-doped with $\mathrm{Yb}$ and Al can be explained in the following way. It is generally known that the main background impurities in GaAs are oxygen and silicon [5]. It is for epitaxy from Ga melts that conductivity type of the epitaxial layers depends on the temperature of the process and silicon concentration in the melt. At $700-800{ }^{\circ} \mathrm{C}$, which corresponds to the conditions of our experiments, silicon mainly occupies vacant sites in Ga sub-lattice and acts as a donor [7]. Oxygen also acts as a donor in GaAs [7]. Incorporation of the $\mathrm{Yb}$ into the melt causes interaction of ytterbium with silicon and oxygen [8] accompanied with creation of slow-moving complexes in the solution-melt. Due to that, amount of these elements entering the epitaxial layer becomes lower - in other words, "cleaning" occurs. Codoping of the solution-melt with $\mathrm{Yb}$ and Al slightly changes the behaviour of the redistribution of the background impurities. Aluminium being added into the melt occupies $\mathrm{Ga}$ vacancy sites, because $\mathrm{Al}$ covalent radius ( $1.24 \AA)$ is very close to Ga one (1.25 $\AA$ ) [9]. Due to that, the amount of Si that also occupies Ga vacancy sites during crystallisation sufficiently decreases. In our opinion, simultaneous action of these two mechanisms, namely, binding of silicon and oxygen in Ga melt on the one hand, and decrease of the probability of entering for Si into the Ga sub-lattice under Al impurity influence on the another hand, results in the anomalous "cleaning" effect of the epitaxial GaAs layers grown from the melts co-doped with $\mathrm{Yb}$ and $\mathrm{Al}$. Due to the reasons mentioned above, increase of Al content decreases the amount of Si entering the epitaxial layer. As a result, conductivity inversion from electron to hole type occurs at lower $\mathrm{Yb}$ concentration in Ga melt when Al concentration increases in it.

\section{Conclusions}

Our investigations showed that co-doping of Ga melts with $\mathrm{Yb}$ and $\mathrm{Al}$ during LPE growth of the epitaxial layers in the temperature range $800-700{ }^{\circ} \mathrm{C}$ enables to obtain materials with electrophysical parameters close to the theoretically predicted ones for undoped layers. Effective "cleaning" of the doped layers from the background impurities can be explained by simultaneous $\mathrm{Yb}$ gettering action with respect to the oxygen and silicon in the solution-melt, and also by lowering the concentration of $\mathrm{Si}$ in the films due to $\mathrm{Al}$ entering into Ga sublattice. This result opens possibilities to create low-doped uncompensated GaAs and AlGaAs layers suitable to produce high-voltage diodes, detectors and thermosensors with improved operation characteristics.

\section{References}

1. V.A. Bespalov, A.G. Yolkin, B.G. Zhurkin, F.I. Kvit, S.R. Oktyabr'sky, G.A. Perezhogin. Mechanism of rare-carth element influence on properties of GaAs layers grown with liquid-phase epitaxy // Short notes in physics, №9, p. 32-34 (1987), in Russian.

2. J. Novotny, O. Prochazkova, K. Zdansky, J. Zavadil. Preparation and properties of $\mathrm{Er}$ and $\mathrm{Yb}$ doped In-based semiconductor compounds // Czechoslovak Journal of Physics. 49(5), p. 757-763 (1999).

3. D.M. Zayachuk, S.I. Krukovsky. Influence of rare-earth elements $\mathrm{Gd}$ and $\mathrm{Yb}$ impurities on electrophysical properties of epitaxial GaAs layers // Visnyk Natsional'nogo Universitetu "Lvivs'ka Politekhnika", №430, p. 73-75 (2001), in Ukrainian.

4. V.I. Korol'kov, N. Rakhimov. Diodes, transistors and thyristors based on heterostructures. "FAN", Tashkent, 1986, 152 p. (in Russian).

5. V.B. Ufimtsev, R.Kh. Akchurin. Physico-chemical bases of liquid-phase epitaxy. "Metallurgiya", Moscow, 1983, 224 p. (in Russian).

6. S. Sze. Physics of semiconductor devices. "Mir", Moscow, 1984, $456 \mathrm{p}$.

7. F.S. Shishiyanu. Diffusion and degradation in semiconductor materials and devices. "Shtiintsa", Kishinev, 1978, 230 p. (in Russian).

8. V.A. Lebedev, V.I. Kober, L.F. Yamshchikov. Thermochemistry of rare-earth and actinoid element melts. Handbook, "Metallurgiya", Chelyabinsk, 1989, 223 p. (in Russian).

9. S.S. Stral'chenko, V.V. Lebedev. $A^{3} B^{5}$ compounds. Handbook. "Metallurgiya", Moscow, 1984, 137 p. (in Russian). 